\title{
Lo didáctico como expresión de lo curricular. Un acercamiento para comprender las prácticas educativas contingentes y emergentes
}

\author{
CONCEPCIÓN BARRÓN TIRADO* \\ Universidad Nacional Autónoma de México (UNAM) - México \\ DALIA GARCÍA TORRES ${ }^{* *}$ \\ Universidad Nacional Autónoma de México (UNAM) - México \\ Recibido el 11-06-21; evaluado el 11-07-21; \\ aceptado el 30-07-21
}

\section{RESUMEN}

El objetivo de este artículo es compartir las reflexiones generadas en torno a las intervenciones didácticas emergentes de los docentes como una expresión de lo curricular en el contexto de la pandemia originada por la COVID-19. La irrupción de cambios contingentes atravesó las diferentes lógicas institucionales, organizacionales, pedagógicas, curriculares, didácticas y tecnológicas e impactó las formas de pensar de los diferentes agentes educativos, modificando sus condiciones de trabajo. Con base en literatura especializada y algunos reportes de instituciones mexicanas que recuperan la voz de los docentes, se abre un debate en torno a las prácticas curriculares y didácticas construidas y reconstruidas en contextos de incertidumbre.

Palabras clave: didáctica, currículum en proceso, práctica docente, prácticas curriculares.

\footnotetext{
* Doctora en Pedagogía por la Facultad de Filosofía y Letras de la Universidad Nacional Autónoma de México (UNAM). Miembro del Sistema Nacional de Investigadores. Investigadora del Instituto de Investigaciones sobre la Universidad y la Educación (IISUE)/UNAM y profesora del Posgrado en Pedagogía (UNAM). Líneas de investigación: didáctica, curriculum, formación profesional y mercado de trabajo. https://orcid.org/0000-0003-4214-9228. Correo electrónico: barront@unam.mx

** Licenciada en Pedagogía por la Facultad de Filosofía y Letras de la Universidad Nacional Autónoma de México (UNAM). Ha participado en distintos proyectos de investigación en el IISUE /UNAM y la Asociación Francófona Internacional de Investigación Científica en Educación (AFIRSE) Sección Mexicana. https://orcid.org/0000-0003-0930-5727. Correo electrónico: daliagarcia0511@gmail.com
} 


\section{Didactics as an expression of the curricular. An approach to understanding contingent and emergent educational practices.}

\section{Abstract}

This article aims at sharing the reflections produced around the emergent didactic interventions of the teachers as an expression of the curricular, in the context of the COVID-19 pandemic. The irruption of contingent changes came across an array of institutional, organizational, pedagogic, curricular, didactic and technological logics, and made an impact on the mindsets of different educational agents, transforming their working conditions. Based on specialized literature as well as reports of Mexican institutions that record down the teacher's voices, a debate is opened around the curricular and didactic practices, constructed, and reformulated in contexts of uncertainty.

Keywords: didactics, curriculum in process, teaching practice, curriculum practices.

\section{O didático como expressão do currículo. Uma abordagem para a compreensão de práticas educacionais contingentes e emergentes}

\section{Resumo}

O objetivo deste artigo é compartilhar as reflexôes geradas em torno das emergentes intervençôes didáticas de professores como uma expressão do currículo no contexto da pandemia provocada pelo COVID-19. A irrupçáo de mudanças contingentes atravessou as diferentes lógicas institucionais, organizacionais, pedagógicas, curriculares, didáticas e tecnológicas e impactou as formas de pensar dos diferentes agentes educativos, modificando suas condiçôes de trabalho. Com base na literatura especializada e em alguns relatos de instituiçóes mexicanas que resgatam a voz dos professores, abre-se um debate em torno das práticas curriculares e didáticas construídas e reconstruídas em contextos de incerteza.

Palavras-chave: didática, currículo em processo, prática docente, práticas curriculares.

\section{INTRODUCCIÓN}

En el contexto de pandemia originada por la COVID-19 y ante la exigencia sanitaria de evitar el contacto físico, las instituciones educativas se vieron obligadas a continuar sus actividades por vía remota. El cierre de las aulas provocó la migración forzada hacia la virtualidad en todos los niveles educativos, por lo que la emergencia educativa se sumó a la sanitaria, manifestándose la brecha digital, las carencias y las desigualdades sociales, tanto en la disponibilidad 
de recursos como en la sensibilización de profesores y alumnos para transitar hacia una cultura digital y hacia una educación mediada por tecnologías. Este contexto ha significado un momento que obliga a repensar el sentido de las distintas formas de vida, pero, sobre todo, de la institución escolar, del currículo formal, de los contenidos disciplinarios muchas veces alejados de la realidad, de las prácticas docentes hegemónicas y de la gestión académicoadministrativa atada a reglamentos obsoletos (Barrón-Tirado, 2020).

La pandemia ha sido un evento de dislocación en tanto «desajuste muy fuerte que rompe la espacialidad-estructuralidad, a través de la temporalidad, y que por ello es irrepresentable» (De Alba, 2007, p. 54), al tiempo que representa una contingencia que nos presenta múltiples posibilidades. Asimismo, es un marco coyuntural que genera interpretaciones y traducciones realizadas por los profesores en torno al currículum, con el propósito de llevar a cabo la adaptación de los contenidos y la creación de estrategias para lograr que los estudiantes aprendan y valoren sus avances académicos. $\mathrm{Al}$ respecto Casimiro y Oliveira (2021) señalan que la noción de traducción es posible plantearla desde Derrida:

para expresar la dislocación producida en los intentos de "poner en práctica» las propuestas curriculares [...]. Con esa opción, cuestionamos la idea de que la práctica remite solo a un proceso mimético —un simple decir «sín— o a la resistencia - un simple decir «no»-, capaz de eliminar las propias dimensiones políticas o limitar tales dimensiones a la participación (o no) en la producción del texto escrito (p. 86).

Considerando lo anterior, este artículo tiene como finalidad ofrecer un primer análisis en torno a las manifestaciones didácticas generadas en un contexto de pandemia, como una expresión de lo curricular. Para ello, destacamos algunos elementos de la teoría curricular y del campo de la didáctica, así como la noción sobre contingencia como una posibilidad entre una multiplicidad de posibilidades (De Alba, 2007). También recuperamos la voz de los docentes expresada en un primer momento a través de encuestas realizadas por distintas entidades educativas mexicanas. El texto se organiza en cinco apartados, a saber: 1) Repensar lo curricular, 2) Procesos didácticos y currículum, 3) Prácticas curriculares y didácticas en un contexto socio histórico emergente, 4) La pandemia como posibilidad y 5) La voz de los docentes y sus prácticas. A partir de los elementos presentados, pretendemos contribuir a la generación de conocimiento articulado en torno a los acontecimientos derivados de un contexto sociohistórico inédito. 


\section{Desarrollo}

\subsection{Repensar lo curricular}

Pensar y cuestionar el currículum necesariamente nos obliga a recuperar su significado y contrastarlo con la realidad. Asimismo, recuperar el significado de un concepto como currículum nos remite a tratar de comprender el contexto en el que surge. En este sentido, no es intención de este trabajo presentar un análisis extenso y profundo sobre el origen del campo y la teoría curricular, sino recuperar, de manera muy concreta, algunos elementos que nos permitan reflexionar en torno a la práctica didáctica como una expresión de lo curricular. Nuestro propósito es dar cuenta de cómo algunos acontecimientos, surgidos en determinados contextos coyunturales, devienen en grandes cambios educativos, que modifican las formas de vida, de ver y de ser en el mundo, así como las concepciones que construimos para explicar la realidad siempre compleja y cambiante.

Distintos autores (Díaz-Barriga, 1989, 1994; Furlán, 1997; Pinar, 2014) coinciden en señalar que la emergencia del currículum como un campo de estudio está relacionada con los acontecimientos sociales, políticos y económicos de la primera mitad del siglo XIX en Estados Unidos; un periodo en el que la sociedad estadounidense se industrializó. Muchas personas migraron del campo a la ciudad, modificando sus formas de vida; ello obligó a cambiar «las estructuras internas de sus escuelas y requirió una revisión de las prácticas pedagógicas vigentes» (Díaz-Barriga, 1994, s.p.). En este periodo de cambio, los trabajos de Dewey, Bobbit y Charter alimentaron las aspiraciones de conformar una sociedad democrática, en el marco de la filosofía pragmática (Díaz-Barriga, 2002). Hacia la década de 1950, y en el marco del contexto de la Guerra Fría (1947-1991), un evento histórico importante tuvo repercusiones significativas en la esfera de lo curricular:

El lanzamiento del satélite soviético Sputnik en 1957 generó muchas dudas sobre la calidad del sistema educativo en los Estados Unidos. [...] El Sputnik generó una obsesión curricular persistente por la ciencia y la tecnología. Imponer tal estandarización curricular significó arrebatar la posibilidad del desarrollo del curriculum a los maestros y a los especialistas de las universidades [...] A esto siguió un manifiesto del curriculum para estructurar el Movimiento de Reforma Nacional del Curriculum a comienzos de los años 60, con el libro El Proceso de la Educación de Jerome Bruner (1960). [...] Bruner esbozó una teoría del curriculum fundamentada en la noción de estructura disciplinar (Pinar, 2014, p. 68) ${ }^{1}$.

1 Las negrillas son propias. 
Las ideas norteamericanas en torno a la teoría curricular migraron a toda América Latina a través de los mecanismos de aculturación (contacto cultural de la teoría curricular originada en E.U. por medio de la traducción de textos y su lectura por intelectuales latinoamericanos), hibridismo (mestizaje o fusión generado en el marco de contradicciones y desigualdades de las naciones latinoamericanas) y cosmopolitismo (una postura de apertura, diversidad y convivencia de culturas en la experiencia individual, bajo la voluntad de comprometerse con el «otro») permeando la forma de ser y hacer currículum en nuestras instituciones educativas (García-Garduño, 2011). Considerando dichos mecanismos, recuperamos referentes latinoamericanos y de otras latitudes, con la intención de ofrecer respuestas a los retos que la realidad nos plantea. Bajo esta perspectiva, destacamos que hacia la década de los ańos noventa del siglo pasado, un momento de grandes aportaciones desde América Latina para el campo, Alicia de Alba define una noción en torno a lo curricular que sin duda sigue siendo un referente vigente:

Por curriculum se entiende a la síntesis de elementos culturales (conocimientos, valores, costumbres, creencias, hábitos) que conforman una propuesta político-educativa pensada e impulsada por diversos grupos y sectores sociales cuyos intereses son diversos y contradictorios, aunque algunos tiendan a ser dominantes o hegemónicos, y otros tiendan a oponerse y resistirse a tal dominación o hegemonía. Síntesis a la cual se arriba a través de diversos mecanismos de negociación e imposición social. Propuesta conformada por aspectos estructurales-formales y procesuales-prácticos, así como por dimensiones generales y particulares que interactúan en el devenir de los currículos en las instituciones sociales educativas. Devenir curricular cuyo carácter es profundamente histórico y no mecánico y lineal. Estructura y devenir que conforman y expresan a través de distintos niveles de significación (De Alba, 1994, p. 59) ${ }^{2}$.

Para De Alba esta síntesis de elementos culturales se articula a partir de tres dimensiones: 1) la dimensión de la sobre determinación curricular, 2) la dimensión estructural-formal y 3) la dimensión de los procesos y prácticas curriculares. La sobre determinación es un concepto cuyo origen se encuentra en la lingüística y en la psicología (Laclau y Moufre, 1987, p. 163) y es recuperado por De Alba para aludir al sentido simbólico del currículum, a esa síntesis de elementos culturales aterrizados en una propuesta político-educativa en tanto que a través de ella se generan contornos sociales, es decir, se define el deber ser de la educación de acuerdo con la idea de ciertas formas de vida. Por su parte, la dimensión estructural formal refiere a los planes y programas de estudio, y la

\footnotetext{
2 La negrillas son propias.
} 
dimensión de los procesos y prácticas aterriza en lo acontecido en el aula a partir de la puesta en marcha de los «aprendizajes curriculares» (Díaz-Barriga, 1997).

Existen otras concepciones sobre currículum complementarias que interesa enfatizar: 1) currículum como verbo (currere), como una acción que invita a la autorrealización, para reconstituir la esfera subjetiva y por ende la social (García-Garduño, 2014), 2) currículum como experiencia a desarrollar en la escuela o como conjunto de experiencias de aprendizaje —currículum como acción-, 3) currículum como plataforma para la descripción y mejora de la realidad de las clases y para la reconstrucción del conocimiento configurador de la práctica — currículum como representación de la acción- (Ferrández, 1992), y 4) el currículum vivido como oportunidad de los maestros para hacer currículum, lo que desencadena un proceso artesanal de modificación curricular (Angulo, 2017).

Dichas concepciones aluden a una visión de los docentes como agentes activos que adquieren conocimientos y estrategias en el proceso de desarrollo del currículum y no solo son consumidores y/o ejecutores de este. Sin importar el papel que asuma en el proceso de diseñar la práctica, el profesor es un «agente activo en la concreción de los contenidos y significados de los currículos, moldeando a partir de su cultura profesional cualquier propuesta que a él se le haga» (Gimeno-Sacristán, 1989, p. 124), por lo que se le puede considerar como un "traductor» que participa en la configuración de los significados de las propuestas curriculares, mismas que no son aisladas ni neutras, son reflejo de un contexto social determinado.

De esta manera, es posible observar que el currículum converge con lo didáctico en el aula (sea presencial o virtual), un momento y espacio en el que la dimensión estructural formal del currículum y la dimensión de los procesos y prácticas se articulan a partir de un plan de estudios, el cual representa un dispositivo que concentra la selección y organización de un determinado contenido, cuya finalidad es la de ser enseñado por los profesores, quienes a su vez lo interpretan, lo traducen, construyen y reconstruyen para compartirlo con los alumnos, al tiempo que integran metodologías de enseñanza que prueban, reproducen, crean y recrean.

La integración de Didáctica y Currículum se produce en una concepción del currículum como un proceso... diferenciar entre currículum y enseñanza puede tener sentido para tratar específicamente los problemas planteados a cada nivel, pero una comprensión global de la realidad escolar exige su integración (Bolívar, 2008, p. 77) . $^{3}$

3 La negrillas son propias. 
Pensar el currículum como proceso implica tener en cuenta que lo prescripto no se conserva de manera estable, inmóvil, sino que se encuentra en movimiento. En este sentido, para Terigi (1994) los procesos curriculares se dan de dos formas: 1) como procesos de concreción y 2) como procesos de disolución. Pensar el currículum como proceso de concreción implica pensar que, a nivel político, institucional y del aula, la prescripción de la enseñanza se mantiene con pocas o ninguna modificación; lo prescrito solo cambia si es necesario para que este logre realizarse. En cambio, pensar el currículum como un proceso de disolución plantea la idea contraria: en el proceso curricular, la prescripción tiende a diluirse y lo que sucede en el aula es lo que en rigor constituye el currículum.

\subsection{Procesos didácticos y currículum}

Al igual que la teoría curricular y el currículum, la didáctica es una disciplina determinada por el contexto; además, es imprescindible entender que la didáctica no se limita a su dimensión técnica, sino que responde a «proyectos sociales y coyunturales históricos. Estos procesos sociales establecen diversas formas de presión para que la escuela se ajuste a un momento histórico en lo particular» (Díaz-Barriga, 1995, p. 9). Desde Comenio en el siglo XVII hasta el momento actual, las diversas posturas didácticas coexisten de manera no ortodoxa, dependiendo de la época y de los proyectos político-educativos se privilegian algunas en detrimento de las otras, o bien se hacen combinaciones por momentos contradictorias (Díaz-Barriga, 1998).

La didáctica como disciplina va reinventando su sentido en el tiempo y de contexto a contexto, con cierta distancia entre el ámbito académico de producción y los sistemas escolares; pero, aunque contradictoria, remite una y otra vez a la enseñanza, a esa intención política, legítima, siempre renovada, de crear dispositivos para dejar huella, para ejercer influencia (Coria, 2016, s.p.).

Asimismo, la didáctica puede ser concebida como una ciencia que se estructura con base en "algunos supuestos básicos, hipótesis y conceptos comunes a más de una teoría científica y centrada en una peculiar definición de su objeto de conocimiento y de acción: la enseńanza [...] como actividad que tiene como propósito principal la construcción de conocimientos con significado» (Litwin, 1997, p. 43). Asimismo, no hay que perder de vista que la enseñanza como una práctica docente que se diseña, se crea y recrea en el aula, es una práctica multideterminada por la institución, por el proyecto educativo, por el currículum y por el conocimiento profesional; además, se asocia a las concepciones docentes conformadas a partir de las creencias, significados, conceptos, así como de los conocimientos disciplinarios, pedagógicos 
y didácticos; todos ellos permean la manera de percibir la realidad y las prácticas que desarrollan en el aula.

Aunado a lo anterior, la enseñanza presupone una posición ideológica y una visión particular de la disciplina, imbuida de las creencias y saberes construidos a lo largo de la trayectoria profesional, por lo que definir las prácticas de enseñanza implica recuperar la ética y los valores «inherentes a la condición humana, pero desde sus actores en los ámbitos escolares» (Litwin, 1997, p. 94). La didáctica, como un saber especializado de la enseñanza, constituye un saber complejo que se desarrolla en el hacer cotidiano de las prácticas docentes ejercidas a través de las interrelaciones subjetivas que entablan con los estudiantes, en contextos culturales, sociales e institucionales normativos, polémicos y contradictorios.

La docencia ligada a una visión sobre lo didáctico, al conocimiento teóricopráctico y a la actividad reflexiva sobre la práctica, puede comprenderse como un fenómeno complejo por la multiplicidad de factores que la determinan y por su naturaleza siempre dinámica y en muchos sentidos impredecible (Gimeno-Sacristán, 1989). La didáctica como el lugar de convergencia entre las propuestas teóricas curriculares y las prácticas educativas, lejos está de constituirse en un espacio de certezas absolutas; las decisiones que el docente toma en cuenta para la selección y organización de las experiencias de aprendizaje no se reducen a una mera elección de corte técnico, sino que implica una reconstrucción compleja teórico-práctica que se realiza con el propósito de que los alumnos aprendan, lo que constituye el principal objetivo.

Esto significa, por parte de los docentes, una reconstrucción de las relaciones entre los contenidos [curriculares] desde la problemática del aprender. La utilización de los medios incluidos en las estrategias metodológicas constituye, en el caso de las prácticas universitarias, resoluciones personales que dan cuenta del pensamiento del docente en relación con la construcción del conocimiento (Litwin, 1997, p. 65).

En el ámbito de la didáctica, los contenidos y los métodos constituyen dos dimensiones clásicas e indisociables para abordar el conocimiento en las aulas. En este sentido, se puede reconocer la libertad que tiene el docente de recrear «el método» en el aula, de recrear los contenidos y las formas de abordarlos, en función de sus concepciones acerca de la didáctica: «nadie es docente, ni aprende a serlo en abstracto. Ser docente es siempre una historia personal, no solo porque es el reflejo de un recorrido singular, sino porque se hace siempre en relaciones concretas, con estudiantes singulares, en situaciones concretas» (Contreras y Pérez de Lara, 2013, p. 257). Cada profesor despliega una manera 
particular para favorecer los procesos de construcción del conocimiento, nos referimos a lo que Litwin (2000) denomina "configuraciones didácticas», una manera particular que el docente despliega:

para favorecer los procesos de construcción del conocimiento. Esto implica una construcción elaborada en la que se pueden reconocer los modos en que el docente piensa su campo disciplinario y que se expresa en el tratamiento de los contenidos, el particular recorte de los mismos, los supuestos que maneja respecto del aprendizaje, la utilización de prácticas metacognitivas, los vínculos que establece en la clase con las prácticas profesionales involucradas en el campo de la disciplina de que se trata, el estilo de negociación de significados que genera, las relaciones entre la práctica y la teoría que involucran lo metódico y la particular relación entre el saber y el ignorar (Litwin, 2000, p. 10).

La noción de configuración didáctica posibilita el reconocimiento de la puesta en juego de las concepciones epistemológicas del profesor en torno al conocimiento, a lo didáctico, a su práctica, a la enseńanza, al aprendizaje, al papel que juegan los contenidos en la construcción del conocimiento y a la relación docente-alumno. Al observar la realidad desde una perspectiva macro, generalmente se tiende a invisibilizar las prácticas de los actores del currículum, lo que deja de lado la singularidad de los procesos, las negociaciones y las resistencias que ocurren en la cotidianeidad. Por ello, resulta imprescindible aceptar la invitación a analizar el currículum a la luz de la práctica didáctica como posibilidad de cambios significativos. No olvidemos que el campo del currículum está sujeto a «un debate siempre vivo, inacabado y escurridizo, porque refleja el carácter abierto, plural y cambiante de la sociedad y de la cultura» (Gimeno-Sacristán, 2010, p. 36).

Como campo práctico complejo, la didáctica incluye una gama de actividades que van desde la planeación, puesta en marcha, experimentación, evaluación, crítica, defensa y reconstrucción. Incluye también los recursos materiales y tecnológicos que la configuran. Por lo que se conforma de múltiples tipos de prácticas, ya sea políticas, administrativas, de evaluación y creación intelectual, entre otras, todas ellas inciden en la acción pedagógica y representan procesos artesanales de modificación curricular (Angulo, 2017). Su valor real está en función de los contextos en los que se desarrolla y cobra significado.

\subsection{Prácticas curriculares y didácticas en un contexto sociohistórico emergente}

Ante la situación sanitaria, los docentes tuvieron que adaptar sus condiciones de trabajo para comunicarse con los estudiantes vía remota. La irrupción de 
los cambios atravesó las diferentes lógicas institucionales, organizacionales, pedagógicas, curriculares, didácticas y tecnológicas e impactó las formas de pensar y actuar por parte de los diferentes agentes educativos. Dichas situaciones permitieron prefigurar nuevas coreografías didácticas (Cid, 2009) en la búsqueda de lograr no solo concretar un currículum, sino la autonomía de sí mismos y de los estudiantes.

Durante este periodo, las TIC han ocupado un papel protagónico y los profesores se han visto obligados a reinventarse para llevar a cabo las clases en aulas virtuales. No obstante, el saber utilizar las tecnologías no es sinónimo de saber enseñar con y desde ellas, además de la necesidad de tomar en cuenta los diferentes contextos tecnológicos de los estudiantes. Por otra parte, es imprescindible reconocer que la pandemia y las tecnologías han trastocado las prácticas curriculares, sin acabar de comprender sus alcances y significados.

A pesar de contar con un abundante trabajo de investigación que promueve enfoques curriculares que abogan por la participación activa de sus actores, al contrastar la práctica dada en un contexto de pandemia, es posible darnos cuenta de que domina el enfoque curricular centrado en los contenidos. En el marco del contexto emergente, se ha pretendido seguir con las mismas lógicas de trabajo embebidas por las culturas heredadas, preservando, diríamos en el lenguaje de Foucault, un determinado régimen de verdad y de autoridad cultural en el que se privilegian ciertos contenidos y formas de conocimiento sobre otros (Dussel, 2014).

Los supuestos pedagógicos didácticos de la escolarización, planteados por la escuela en la modernidad con la aspiración de atender a grandes poblaciones y cumplir con la encomienda del estado moderno de educar a toda la población, a partir de la estandarización académica-administrativa, de la regulación de los planes de estudios, de los espacios físicos, y de las prácticas de los agentes del currículum (Terigi, 2015), han sido trastocados, así como sus principios, tales como el de simultaneidad, presencialidad, gradualidad y el cronosistema (Terigi, 2020).

En relación con el principio de simultaneidad, se intenta enseñar el mismo orden del saber a todos, una estrategia de la escolarización de la educación de masas y ahorro de recursos, a través de diversas plataformas digitales: se ha procurado atender al mayor número de estudiantes, enfrentándose a la falta de recursos tecnológicos y de conectividad de la población. En cuanto a la presencialidad, pensada para que la transmisión del saber ocurra por copresencia, manteniendo un control y seguimiento puntual de los estudiantes, se ampliaron las posibilidades de trabajo ya fuera de forma síncrona o asíncrona. Asimismo, el cronosistema de los contenidos y de la duración de la jornada de 
trabajo se exacerbó ante la falta de relación entre los acontecimientos vividos y los temas establecidos en el currículum, transmitidos por vía remota.

El saber pedagógico, circunscrito por ańos para apoyar el funcionamiento homogéneo y estandarizado del sistema escolar, de la población, de los aprendizajes y de las prácticas educativas, fue cuestionado y relativizado por los docentes ante la contingencia educativa, quienes se vieron en la necesidad de responder a nuevos códigos, nuevos marcos de referencia, haciendo uso de sus saberes y de su creatividad didáctica. Los docentes se han apropiado de las TIC de diferente manera y las utilizan según sus necesidades, no obstante, algunos otros han mostrado resistencia, por lo que se visibiliza la urgencia de generar una cultura digital amigable que permita transitar hacia nuevas posibilidades de uso didáctico.

Es importante no soslayar la compleja interrelación entre lo pedagógico, lo tecnológico y lo disciplinar a la hora de incorporar las TIC en el aula, así como aprender a enseńar con tecnología, a partir de una práctica didáctica creativa generada por un docente intelectual, para contextos reales de enseńanza y de aprendizaje, pero, sobre todo, una didáctica creada para dar respuesta a un contexto sociohistórico emergente.

\subsection{La pandemia como posibilidad}

Es conveniente destacar que, en un sentido estricto, el termino contingencia alude a la posibilidad de que algo suceda (RAE, 2021). Por su parte, De Alba (2007, p. 55) nos plantea la contingencia como «la elección de una posibilidad entre una multiplicidad de posibilidades» y es un asunto que se vincula directamente con la toma de una decisión, además de trastocar la noción de historia y de progreso «en la medida en que, por medio de la contingencia, una de las posibilidades se activa como constitutiva de la realidad, de una identidad, de un proceso" (De Alba, 2007, p. 55). La declaratoria de una pandemia a causa de la COVID-19 sin duda es un evento contingente en tanto nos presenta diversas y múltiples posibilidades de ser, nos activa como sujetos que toman decisiones, al tiempo que se activan diversas realidades potenciales.

Sumado a lo anterior, se puede señalar que el sistema educativo, la institución y el currículum escolar constituyen un espacio de poder y de toma de decisiones, en los que el conflicto, las contradicciones y los dilemas permean las prácticas de los diversos grupos que ostentan diversas posiciones; esta configuración persiste aun cuando la escuela, como institución-estructura sólida, pero sedimentada y hasta cierto punto inmóvil (De Alba, 2007), ha sido dislocada por la pandemia. Todas las comunidades, desde la educación básica hasta la 
educación superior, de todo el mundo, modificaron sus formas de vida: transitaron de la presencialidad a una educación remota, de manera abruptay en medio de cierto caos e incertidumbre; la única certeza fue tener un currículum oficial determinado, prescrito, que debía cumplirse como promesa (Casimiro, 2008).

Ante la pandemia, el imperativo de las políticas educativas globales fue la incorporación de las tecnologías y de la cultura digital, no solo como medida emergente, sino como un eje estructurante de un proyecto educativo. Si bien, este imperativo fue compartido por los gobiernos de cada país, el desarrollo de sus significados en cada contexto local fue diferido, generando distintas posibilidades y directrices para dar continuidad al trabajo en las instituciones educativas. Cabe señalar que las políticas, concebidas como procesos, sujetas a interpretaciones por parte de las instituciones y de los actores, orientaron las formas creativas en que fueron puestas «en acto» (más que «implementadas») (Ball, 1993; Beech y Meo, 2016), lo que ha implicado tomar en cuenta el dinamismo del contexto para encontrarle sentido a una realidad empírica inédita que está en permanente cambio; una perspectiva distinta a la de solo considerar la medición de su impacto, bajo una visión estática y monolítica de la realidad.

La puesta en acto de las políticas educativas y de cómo los actores del currículum le otorgaron un sentido, las cuestionaron, las redefinieron, las desconocieron o tradujeron, dentro de los límites y posibilidades de las formaciones discursivas generadas en este periodo, alude a un posicionamiento crítico-reflexivo del acontecimiento pandémico, como «el momento dislocador y disruptivo en el cual los fundamentos se derrumban» (Marchart, 2009, p. 15). Por su parte, Ottoni (2003) sostiene que, en la traducción comprendida como deconstrucción, no existe fidelidad a determinado origen, sino responsabilidad en la tarea de traducir lo intraducible.

El proceso de significación del currículo, desarrollado a través de una negociación llevada a cabo por los profesores durante un acontecimiento pandémico, ha generado diversas interpretaciones y prácticas en relación con la incorporación de las tecnologías, que de manera abrupta contribuyeron a la dislocación del contexto escolar hasta entonces conocido. Así, es posible avizorar un momento histórico que propicia una abundante expresión de lo curricular a través de procesos didácticos, en tanto los docentes asumieron mayor posibilidad de decidir qué enseñar, creando y recreando formas de hacer y conocer, independientemente de los fundamentos teóricos y las formas de gestión o diseño curricular; ello fue potenciado con la incorporación de las tecnologías de la información y la comunicación (TIC), pues dadas sus características, permiten un sinfín de posibilidades didácticas, lo que representa una oportunidad de cambio educativo significativo. 


\subsection{La voz de los docentes y sus prácticas}

¿En dónde es posible observar lo didáctico como expresión de lo curricular? En un primer acercamiento recuperamos las voces de los docentes y sus prácticas a partir de los reportes generados por las encuestas aplicadas por diferentes instituciones educativas en México. Si bien las encuestas proporcionan información mínima en torno a las diferentes estrategias didácticas seguidas para llevar a cabo el currículum formal, representan una primera base para identificar los procesos de traducción, interpretación y puesta en práctica del currículum a partir de las estrategias didácticas generadas. En dichas encuestas podemos encontrar visos de nuevas configuraciones didácticas, como expresiones de lo curricular, construidas en situaciones de contingencia, lo que permite problematizar no solo en torno a lo que sucede en el presente, sino sobre la gama de posibilidades de cambio.

Para ejemplificar lo anteriormente expuesto, iniciaremos con la información obtenida por la Comisión Nacional para la Mejora Continua de la Educación (MEJOREDU), a través de la aplicación de una encuesta a estudiantes, madres y padres de familia, docentes y directores de escuelas de educación básica (EB) durante junio de 2020. Específicamente, con los docentes se indagó acerca de las estrategias para dar continuidad a la enseńanza y el aprendizaje, los apoyos recibidos por la escuela, los recursos para enseñar desde el hogar, la comunicación entre estudiantes, familia y escuela. El 99\% de los docentes encuestados reportó haber continuado sus clases con el apoyo de las estrategias y medios ofrecidos por la Secretaría de Educación Pública (SEP), con estrategias adicionales y con aquellas que diseńaron individualmente, enfrentando serios problemas por parte de los estudiantes por no contar con los dispositivos electrónicos para trabajar. Sin embargo, un 46,3\% reconoció que los contenidos televisivos creados exprofeso para enfrentar la contingencia no fueron suficientes para que los estudiantes pudieran seguir aprendiendo, pues los contenidos estaban en una lengua diferente de la que hablaban sus estudiantes (26,8\%) o los estudiantes no contaron con sus libros de texto durante la contingencia (20\%), entre otras causas (MEJOREDU, 2020, p. 33).

A partir de los resultados de esta encuesta se realizaron algunas sugerencias para diseñar estrategias para la atención diferenciada por nivel y servicio educativo, considerando las principales desigualdades de los estudiantes y sus familias, en la búsqueda de establecer un currículo de emergencia basado en la priorización y flexibilización de contenidos, a la vez de ofrecer al personal docente y directivo formación pertinente y de calidad para situaciones de emergencia. 
Por su parte, en la Universidad Nacional Autónoma de México (UNAM) se aplicaron dos encuestas con la finalidad de contar con información pertinente y actualizada acerca de los retos a los que enfrentaban los profesores de bachillerato, licenciatura y posgrado para realizar su labor cotidiana en línea. La primera fue levantada el 25 de marzo de 2020 y se reporta que, de 383 profesores, el 60,5\% estuvo de acuerdo con el apoyo institucional brindado por la UNAM durante la contingencia. El 43,3\% enfrentó problemáticas de tipo logístico, 39,7 \% fueron de tipo tecnológico, 35,2\% de tipo pedagógico y $14,9 \%$ de tipo socioafectivo. La comunicación se entabló principalmente desde correos electrónicos, Facebook y WhatsApp. El trabajo académico se llevó a cabo principalmente a través de Google Classroom, Moodle, Google Suite, Zoom, Google Hangouts y Skype. La mayoría indicó requerir de apoyo tecnológico y asesoría didáctica, así como de cursos relacionados con formación pedagógica, disponibilidad de recursos digitales, recursos informativos sobre uso de tecnologías en educación, mejora de procesos institucionales, acompañamiento pedagógico, asesoría técnica e información de los recursos institucionales (Sánchez et al., 2020).

El 17 de junio de 2020 la UNAM aplicó una segunda encuesta con el propósito de explorar las condiciones pedagógicas, experiencias didácticas y de evaluación a las que se enfrentaron los docentes durante este periodo. Los resultados reflejan que, de 513 profesores que dieron respuesta, el 43\% mantuvo el esquema de su práctica presencial, trasladándola solo al espacio virtual, el 23\% indicó que su práctica mejoró respecto al trabajo presencial y el 34\% considera que su práctica empeoró. El 76\% empleó el correo electrónico como herramienta de interacción docente-estudiante $y$, antes que usar WhatsApp como herramienta, los docentes dicen utilizar con mayor frecuencia plataformas como Moodle, Google Classroom u otra (53\%) y las videoconferencias (51\%). Asimismo, los docentes se interesaron por cursos sobre diseño de situaciones didácticas para entornos no presenciales (75\%), cursos sobre instrumentos y estrategias de evaluación para entornos no presenciales (73\%) y usos educativos de aulas virtuales (69\%) (Sánchez y Martínez, 2020).

Por su parte, el Consejo Mexicano de Investigaciones Educativas (COMIE), el Centro de Investigación y de Estudios Avanzados (Cinvestav), la Unión de Universidades de América Latina (UDUAL) y la Red de Cátedras Unesco difundieron un instrumento sobre educación superior realizado por la red de investigación «Educación, investigaciones y actualidades» (EDRAC, por sus siglas en francés). Se recibieron 301 respuestas de docentes de instituciones públicas y privadas, entre el 27 de abril y el 7 de mayo de 2020, destacándose que los docentes se preocupan por sus estudiantes 
en tanto sujetos de aprendizaje, pero, asimismo, ciudadanos con derechos iguales, independientemente de los sesgos en sus posiciones sociales y económicas. Les inquietan en particular las desigualdades en el acceso al conocimiento, digitalmente suministrado, de los alumnos que no disponen de las herramientas tecnológicas para asistir a las clases virtuales y de cuya fragilidad académica previa les impide sacar provecho de ellas. El dejar atrás a esos grupos y el no contar con los insumos para retenerlos son fuentes de malestar (Didou, 2020, s.p.).

La información recabada en las diferentes encuestas da por sentado que la continuidad se centra en el uso y manejo de las tecnologías; sin embargo, también reportan que un alto porcentaje de docentes tiende a reproducir estrategias y mecanismos presenciales en entornos virtuales y que posiblemente no se lleve a cabo una reflexión sistemática y crítica en torno a las prácticas emergentes. A pesar de ello, también se observa que los docentes no se conforman con los apoyos que han brindado las instituciones educativas, se preocupan y ocupan por formarse, por tomar cursos que les sirvan de apoyo para continuar y mejorar su labor en el momento emergente, reflejando un posicionamiento activo, crítico y reflexivo.

El espacio físico diferenciado, los recursos y artefactos tecnológicos, la conectividad, el tiempo destinado, las formas diversas de capital cultural, la formación previa de los docentes, el manejo de contenidos y el diseño de estrategias didácticas, así como las condiciones domésticas en las que se desenvuelven los docentes, constituyen retos educativos de diversa índole, los cuales han sido asumidos de manera diferenciada por los diversos actores y que, sin duda, vale la pena explorar y recuperar como expresiones didácticas de lo curricular, en el sentido antes mencionado.

\section{Conclusiones}

En pleno siglo XXI es posible afirmar que el desarrollo de la teoría curricular y el campo de la didáctica, continúan siendo objeto de amplias discusiones epistemológicas y disciplinarias, no obstante, desde una visión integral de lo curricular, es posible asumir una posición didáctica contextualizada. Recuperamos aquí la invitación a entender la didáctica como una disciplina que no se restringe a una dimensión técnica-instrumental, sino como un campo que genera conocimiento a través de metodologías que «responden a proyectos sociales y coyunturales históricas. Estos procesos sociales establecen diversas formas de presión para que la escuela se ajuste a un momento histórico en lo particular» (Díaz-Barriga, 1995, p. 9). 
A pesar de las problemáticas identificadas desde la investigación educativa, y reconocidas de primera línea por los diversos actores educativos, era claro que previo a la declaratoria de pandemia teníamos más certezas sobre qué y cómo enseñar, sobre qué contenidos curriculares y cómo enseńarlos. Trasladar de manera abrupta la enseñanza presencial a una virtual provocó una dislocación en la que sin duda los sujetos tomaron relevancia (De Alba, 2007) ya que, ante la alteración de la escuela como estructura física-material articulada en torno a los supuestos de presencialidad, simultaneidad, gradualidad y cronosistema (Terigi, 2020), cobra relevancia aquello que construyeron y reconstruyeron los distintos actores educativos. $\mathrm{Al}$ respecto, recordemos que en «la medida en que una estructura se encuentra más desestructurada las decisiones que se toman no dependen de la estructura, sino de los sujetos sociales que contribuyen, de distintas formas, a la dislocación o a la erosión de la estructura» (De Alba, 2007, p. 54).

El momento social que prevalece derivado de la pandemia es un momento coyuntural en el que domina el uso de las TIC para tratar de subsanar los supuestos de la escolaridad expresados por Terigi (2020), pero sobre todo, es un momento en el que lo didáctico expresa y da cuenta de las formas de enseñanza dominantes, al tiempo que permite observar atisbos de cambios que justamente tratan de construirse para responder a un momento social futuro en el que se avizora un mundo dominado por la cultura digital. Estamos, pues, ante una gran producción de expresiones didácticas que dan cuenta de infinidad de realidades.

También, estamos ante la posibilidad de revisitar la realidad con una visión abierta a cuestionar, a contrastar y a generar nuevas formas de hacer teorías más acordes con las nuevas e inciertas realidades. Es relevante preguntarnos al servicio de quién está el tiempo educativo, qué ideales producimos y reproducimos además de saberes, qué contenidos curriculares u objetos cognoscibles se ponen al servicio de la comunidad educativa, cómo propiciamos la curiosidad por aprender a través de metodologías distintas y cómo establecemos límites con miras al respeto por el otro y por el mundo en el que vivimos.

El papel del docente y de la escuela pública en tiempos de contingencia y dislocación nos obliga a pensar en las diversas maneras de colaborar para la construcción de una sociedad más justa y equitativa. Desentrañar el sentido de la educación, de la escuela pública, del currículum y de la formación y práctica de los docentes, desde una perspectiva crítica, en la situación de crisis en la que vive la humanidad en todos los ámbitos de la vida, representa una posibilidad de desnaturalizar las desigualdades sociales. 
Sin duda, los profesores constituyen un elemento clave para «superar la brecha entre el currículo como intención y los mecanismos para hacerlo operativo» (Stenhouse, 2010, p. 14); si se deja de lado su participación, se corre el riesgo de que la administración educativa renuncie a «hacer del currículum una palanca de transformación de la enseñanza y de formación del profesorado" (Stenhouse, 2010, p. 13), reduciendo su participación a la de ejecutor, en detrimento de una visión del profesor como un intelectual. En la construcción de un orden pedagógico, el maestro ejerce un juicio profesional, toma decisiones, piensa y actúa en función de determinadas exigencias de racionalidad guiado por una razón práctica, en este caso pedagógica (Tardif y Gauthier, 2005). Toda práctica docente implica un compromiso moral para formar a las generaciones del futuro que tomarán decisiones y juicios en medio de situaciones de incertidumbre inevitable (Fullan y Hargreaves, 1999).

Lo anterior ha sido posible observarlo y contrastarlo con la serie de prácticas surgidas durante la contingencia sanitaria. Sin duda, desde la investigación se plantea el gran reto de recuperar todas estas experiencias didácticas y curriculares operadas desde los propios recursos intelectuales de cada docente. No es menor trabajo ni tarea vana, pues en muchas de esas prácticas posiblemente ya se trabajen enfoques alternativos orientados a recuperar el valor liberador de la educación.

\section{REFERENCIAS BIBLIOGRÁFICAS}

Angulo, R. (2017). Discurso(s) curriculares en la educación superior en México. Revista de Investigación Cualitativa, 2(2), 52-67. [Fecha de consulta 9 de mayo de 2021]. Recuperado de http://investigacioncualitativa.com/index. $\mathrm{php} /$ revista/article/view/62

Ball, S. J. (1993). What is policy? Texts, trajectories and toolboxes. Discourse: Studies in the Cultural Politics of Education, 13(2), 10-17. https://doi. org/10.1080/0159630930130203

Barrón-Tirado, M. C. (2020). La educación en línea. Transiciones y disrupciones. En H. Casanova (coord.), Educación y pandemia: una visión académica (pp. 66-74). Ciudad de México: Instituto de Investigaciones sobre la Universidad y la Educación.

Beech, J. y Meo, A. (2016). Explorando el uso de las herramientas teóricas de Stephen J. Ball en el Estudio de las Políticas Educativas en América Latina. Education Policy Analysis Archives/Archivos Analiticos de Politicas Educativas, 24(23), 1-19. https://doi.org/10.14507/epaa.24.2417

Bolívar, A. (2008). Didáctica y currículum: de la modernidad a la postmodernidad. Archidona: Aljibe. 
Casimiro, A. (2008). Articulaciones en las políticas de currículo. Perfiles Educativos, XXX(120), 63-78. [Fecha de consulta 8 de junio de 2021]. Recuperado de https://www.redalyc.org/articulo.oa?id=13211159004

Casimiro, A. y Oliveira, L. (2021). Discursos curriculares para la enseñanza secundaria: el caso del Estado de Bahía, Brasil. En S. Morelli (coord.), Las politicas curriculares. Experiencias encontextos latinoamericanos (pp. 83-106). Rosario, Argentina: Homo Sapiens Ediciones.

Cid, A. (2009). Coreografias didácticas en la universidad: experiencias e innovaciones. Santiago de Compostela, España: Andavira.

Contreras, J. y Pérez de Lara, N. (2013) (comps.), Investigar la experiencia educativa. Madrid: Morata.

Coria, A. (2016). Didáctica. En A. M. Salmerón, B. Trujillo, A. Rodríguez y M. de la Torre (coords.), Diccionario Iberoamericano de Filosofía de la Educación. Ciudad de México: UNAM-FCE. [Fecha de consulta 7 de noviembre de 2020]. Recuperado de https://www.fondodeculturaeconomica.com/dife/ definicion.aspx?l=D\&id $=50$

De Alba, A. (1994). Curriculum: crisis, mito y perspectivas. Buenos Aires: Miño y Dávila Editores.

De Alba, A. (2007). Curriculum sociedad, el peso de la incertidumbre, la fuerza de la imaginación. Ciudad de México: IISUE.

Díaz-Barriga, A. (1989). La lectura de Tyler. Un síntoma de nuestra ignorancia sobre la historia y los procesos educativos. En A. Furlán y M. A. Pasillas (coords.), Ralph Tyler. Lecturas comprometidas a cuarenta años de «Principios básicos del currículo» (pp. 25-40). Ciudad de México: UNAM/Universidad Autónoma de Sinaloa.

Díaz-Barriga, A. (1994). Contexto del surgimiento de la problemática curricular. En El curriculo escolar. Surgimiento y perspectivas. Buenos Aires: Aique Grupo Editor.

Díaz-Barriga, A. (1995). Didáctica: aportes para una polémica. Buenos Aires: Instituto de Estudios y Acción Social-Aique Grupo Editor.

Díaz-Barriga, A. (1997). Didáctica y curriculum. México: Paidós Educador.

Díaz-Barriga, A. (1998). La investigación en el campo de la didáctica. Modelos históricos. Perfiles Educativos, 20(80), 1-23. [Fecha de consulta 28 de octubre de 2020]. Recuperado de https://www.redalyc.org/pdf/132/13208002.pdf

Díaz-Barriga, A. (2002). Currículum: una mirada sobre su desarrollo y sus retos. En I. Westbury (comp.), ¿Hacia dónde va el curriculum? La contribución de la teoría deliberadora (pp. 163-175). Barcelona: Pomares.

Didou, S. (2020). Experiencias profesionales en periodo de confinamiento: la voz de los académicos. Ciudad de México: COMIE. [Fecha de consulta 8 de junio 
de 2021]. Recuperado de http://www.comie.org.mx/v5/sitio/2020/05/13/ experiencias-profesionales-en-periodo-de-confinamiento-la-voz-de-losacademicos/

Dussel, I. (2014). ¿Es el curriculum escolar relevante en la cultura digital? Debates y desafíos sobre la autoridad cultural contemporánea. Archivos Analíticos de Políticas Educativas, 22(24), 1-26. [Fecha de consulta 8 de junio de 2021]. https://doi.org/10.14507/epaa.v22n24.2014

Ferrández, A. (1992). Realidad y perspectivas de la educación de personas adultas. En A. Ferrández y J. M. Puente (coords.), Educación de personas adultas: macrodidáctica (pp. 21-78). Madrid: Diagrama.

Fullan, M. y Hargreaves, A. (1999). La escuela que queremos. Ciudad de México: Amorrortu-SEP-Biblioteca para la Actualización del Maestro.

Furlán, A. (1997). Ideología del discurso curricular. Ciudad de México: Universidad Autónoma de Sinaloa / Universidad Nacional Autónoma de México.

García-Garduño,J.M. (2011) Acculturation, Hybridity, Cosmopolitanism in IberoAmerican Curriculum Studies. En W. F. Pinar (eds.), Curriculum Studies in Mexico. International and Development Education (pp. 137-163). New York: Palgrave Macmillan. https://doi.org/10.1057/9780230337886_7

García-Garduño, J. M. (2014). Estudio introductorio. En W. Pinar, La teoría del curriculum (pp. 11-59), Madrid: Narcea.

Gimeno-Sacristán, J. (1989). El currículum: una reflexión sobre la práctica. Madrid: Morata.

Gimeno-Sacristán, J. (2010). ¿Qué significa el curriculum? En J. Gimeno Sacristán (coord.), Saberes e incertidumbres sobre el curriculum (pp. 21-43). Madrid: Morata.

Laclau, E. y Mouffe, C. (1987). Hegemonía y estrategia socialista. Hacia una radicalización de la democracia. Madrid: Siglo XXI.

Litwin, E. (1997). El campo de la didáctica. La búsqueda de una nueva agenda. En A. Camiloni (coord.), Corrientes didácticas contemporáneas (pp. 75-90). Buenos Aires: Paidós.

Litwin, E. (2000). Las configuraciones didácticas. Una nueva agenda para la enseñanza superior. Buenos Aires: Paidós.

Marchart, O. (2009). El pensamiento político postfundacional. La diferencia politica en Nancy, Lefort, Badiou y Laclau. Buenos Aires: Fondo de Cultura Económica.

MEJOREDU (2020). Experiencias de las comunidades educativas durante la contingencia sanitaria por COVID-19. Educación básica. Cuadernos de investigación educativa. Ciudad de México: Comisión Nacional para la Mejora Continua de la Educación. [Fecha de consulta 8 de junio de 2021]. 
Recuperado de https://editorial.mejoredu.gob.mx/Cuaderno-Educaciona-distancia.pdf

Ottoni, P. (2003), A responsabilidade de traduzir o in-traduzível: Jacques Derrida e o desejo de [la] tradução. D.E.L.T.A., 19 (especial), 163-174. https://doi. org/10.1590/S0102-44502003000300010

Pinar, W. (2014). La teoría del curriculum. Madrid: Narcea.

Real Academia Española (RAE) (2021). "Contingencia» [Fecha de consulta 4 de agosto de 2021]. Recuperado de https://dle.rae.es/contingencia

Sánchez M., Martínez, A. M., Torres, R., De Agüero, M., Hernández Romo, A., Benavides, M., Jaimes, C. y Rendón, V. (2020). Retos de la educación a distancia en la contingencia COVID-19. Cuestionario a docentes de la UNAM. Revista Digital Universitaria. [Fecha de consulta 30 de abril de 2021]. Recuperado de https://www.revista.unam.mx/prensa/retos-educativos-durante-la-pandemia-de-covid-19-una-encuesta-a-profesores-dela-unam/

Sánchez, M. y Martínez, A. M. (coords.) (2020). Transición de los profesores de la UNAM a la educación remota de emergencia durante la pandemia. Informe Ejecutivo. México: Secretaria General/CUAEEID. [Fecha de consulta: 20 de mayo de 2021]. Recuperado de https://cuaieed.unam.mx/descargas/ Informe\%20Ejecutivo_Encuesta_Docentes_UNAM_CUAIEED_VF.pdf

Stenhouse, L. (2010). Investigación y desarrollo del curriculum. Madrid: Morata.

Tardif, M y Gauthier, C. (2005). El maestro como 'actor racional': racionalidad, conocimiento, juicio. En L. Paquay (coord.), La formación profesional del maestro. Estrategias y competencias (pp. 309-354). Ciudad de México: FCE.

Terigi, F. (1994). ¿Qué clase de cosa es el curriculum escolar? En Diseño, desarrollo y evaluación de curriculum. Documento mimeografiado. Buenos Aires: Ministerio de Cultura y Educación.

Terigi, F. (2015). El saber pedagógico ante los desafios de la inclusión educativa. Buenos Aires: UBA-UNGS- Normal 7 CABA, 12 de junio de 2015. [Fecha de consulta 30 de abril de 2021]. Recuperado de https://issuu.com/ danielalalberto/docs/conferencia_terigi_en_armando_trama

Terigi, F. (2020). Escolarización y pandemia. RevCom, (11), e039. https://doi. org/10.24215/24517836e 039 